Disclosure of Interest: None declared

DOI: 10.1136/annrheumdis-2017-eular.4807

\section{AB0555 ANCA VASCULITIS AND CLASSIC CARDIOVASCULAR RISK FACTORS: COINCIDENCE OR CAUSALITY?}

C. Busca, J.J. Ríos, Á. Robles, A. Noblejas, E. Martínez, C. Soto, I. Vives, J.A. Troncoso, L. Bailón, F. Arnalich. Internal Medicine, la Paz Hospital, Madrid, Spain

Background: Unlike other systemic autoimmune diseases (rheumatoid arthritis or systemic lupus erythematosus), the mechanisms involved and the association between ANCA vasculitis with cardiovascular risk factors (CVRF) or cardiovascular events (CVE) are unknown. There may be a phenomenon of "early" atherosclerosis that contributes to an increased cardiovascular risk. This process would not be explained only by the co-existence of the classics CVRF.

Objectives: We reviewed the prevalence of classical CVRF and CVE in a cohort of patients diagnosed with ANCA vasculitis. We analyzed whether the appearance of these factors was prior to or subsequent to the diagnosis of the disease or during its evolution.

Methods: A descriptive cross-sectional analysis of the classic CVRF and CVE was analyzed in a cohort of patients with ANCA positive vasculitis in follow-up in the Autoimmune Diseases Division of a Spanish hospital. The main demographic characteristics, type of vasculitis and the presence of arterial hypertension, type 2 diabetes mellitus (T2DM), dyslipemia, smoking and obesity were reviewed. Likewise we analyzed CVE (heart failure -HF-, acute coronary syndrome -ACS-, stroke or transient ischemic attack -TIA- and peripheral arteriopathy -PA-) and if each factor was presented at the diagnosis of the disease or they appeared during the evolution after starting immunosuppressive treatment.

Results: A total of 35 patients were studied: 21 women (60\%) and the average age was 53 years old. A number of 15 were microscopic polyangiitis, 9 granulomatosis with polyangiitis and 11 allergic granulomatous angiitis. Twenty one patients presented hypertension, 9 of them (42.9\%) developed it after the diagnosis of vasculitis. From 7 patients with diabetes mellitus, 5 of them were before diagnosed with vasculitis. Nineteen presented dyslipemia and 9 of them $(47.4 \%)$ presented lipid alteration during the evolution of vasculitis. Overweight/obesity was evident in 4 of the 11 cases after the diagnosis of vasculitis. Only 5 patients did not have a cardiovascular event. ACS was observed in 3 patients, HF in 2 and PA in 1 patient. There were no cases of TIA or ischemic stroke. Four of them had dyslipidemia ( 3 after diagnosis of vasculitis) $(\mathrm{p}=0.18)$ and 3 had hypertension ( 2 after diagnosis of vasculitis, $p=0.66)$. Three patients were overweight or obese $(p=0.3)$ and two had T2DM $(p=0.2)$, both of them appeared after the diagnosis. Previous history of smoking was observed in 4 of the 5 patients $(p=0.06)$. In 3 patients $(71.4 \%)$ the cardiovascular event was recorded prior to vasculitis diagnosis and only in 2 cases it occurred during the evolution.

Conclusions: This study shows that a high percentage of patients with ANCA vasculitis also presents some type of classic CVRF despite of CVE were not elevated. The diagnosis and treatment of ANCA-positive vasculitis did not statiscally correlate with a greater number of CVE, therefore it would be necessary to carry out studies with a larger number of patients in order to establish conclusions. It is not well defined that weight may have these factors in the prognosis of patients with ANCA vasculitis. These data suggest the need to maintain a close monitoring and therapeutic approach of classic CVRF in this relatively young group of patients.

References:

[1] Cohen Tervaert JW. Best Pract Res Clin Rheumatol. 2013 Feb;27 (1):33-44 Disclosure of Interest: None declared

DOI: 10.1136/annrheumdis-2017-eular.5262

\section{AB0556 INCIDENCE AND RISK FACTORS OF INFECTIONS IN SYSTEMIC NECROTIZING VASCULITIS}

C.E. Pena, M. Pera, C. Costi, G. Lucila, P. Castellani, Y. Nuccetelli, M. Garcia. HIGA San Martin la Plata, la Plata, Argentina

Background: Infections in patients with systemic vasculitis represent one of the main causes of mortality. Risk factors of infection such as corticosteroid use, intensity of immunosuppressive therapy, age, presence of leucopenia, lymphopenia,hypogammaglobulinemia, associated organic involvement, and dialysis dependence have been identified

Objectives: a)To determine the incidence of infection in patients diagnosed with:Polyangeitis with Granulomatosis (GPA), Eosinophilic Polyangiitis with Granulomatosis (EGPA), Microscopic Polyangeitis (PAM) and Panarteritis Nodosa (PAN), b) clinical characteristics and associated risks factors.

Methods: Analytical, observational,retrospective study. Data source:clinical records of patients diagnosed with ANCA associated vasculitis and Panarteritis Nodosa, evaluated in a center of rheumatology (2000-2016). Variables:Demographic data, clinical manifestations, laboratory data, infectious events serious (requiring hospitalization or prolonged antibiotic/antiviral treatment, recurrences of herpes zoster virus or opportunistic infections), sites of infection, isolated microorganisms, mortality related to the infectious event

Results: 80 patients, $61.25 \%$ women. Mean age at diagnosis: 49.2 years (range 18-77). Types of vasculitis: $41.2 \%$ GPA, $18.7 \%$ EPGA, $26.25 \%$ PAM, $3.73 \%$
PAN not associated with HBV and 10\% ANCA-associated vasculitis that did not met classification criteria. Systemic involvement $(68 \%)$, pulmonary $(59 \%)$, renal $(58 \%)$ and otorhinolaryngology (43.6\%) were the most frequent. 36 infectious events were recorded in 28 patients. Follow-up time: Median 22 m (IQR664). Incidence of infection:38.4\%, with a median of $3 \mathrm{~m}$ (IQR 1-18 m) from diagnosis of vasculitis. Low respiratory infections (40.7\%), sepsis $(39.3 \%)$, and urinary tract infections (15\%) were the most common. $25 \%$ of these patients presented a second infectious event, being low respiratory tract the most frequent site (47\%). Two patients had a 3rd event (soft tissue infection, septic shock). Bacterial etiology was the most prevalent (45\%).Mortality at the 1 st event was $14.3 \%$ (n: 4$) .71 .4 \%$ of patients were in the induction phase of treatment. Immunosuppressants used prior to infectious event: cyclophosphamide (48.1\%), azathioprine $(11.1 \%)$, methotrexate $(7.4 \%)$, mofetil mycophenolate $(3.7 \%)$, none $(22.2 \%)$. Corticosteroids $\geq 30 \mathrm{mg} / \mathrm{d}$ were observed in $35.7 \%$ patients, ranging from $7.5-30 \mathrm{mg} / \mathrm{d}(10.7 \%)$, and $\leq 7.5 \mathrm{mg} / \mathrm{d}$ in $35.7 \%$. Presence of leukopenia (26\%), lymphopenia (44\%), hypoalbuminemia (24\%), renal insufficiency $(63 \%)$ and dialysis dependency (37\%) were identified in patients with infectious events. Renal involvement ( $\mathrm{p} 0.01)$ and dialysis dependence ( 0.001$)$ were significantly associated with infection.

Conclusions: The incidencia of infection was 38.4\%.Lower airway infections, septicemia and urinary tract infections are the most commonly implicated sites. Most infections occurred in the induction phases of the disease. Dialysis dependence and presence of renal involvement were significantly associated with the presence of infection.

References:

[1] Debouverie O.Infections Events During the Course of Systemic Necrotizing Vasculitis A retrospective study of 82 cases. La Revue de Medicine Interne 2010; 35: 636-642.

[2] Kronbickler A. Frecuency, risk factors and prophylaxis of infection in ANCAassociated vasculitis. Eur J Clin Invest 2015; 45 (3): 346- 368.

Disclosure of Interest: None declared

DOI: 10.1136/annrheumdis-2017-eular.1758

\section{AB0557 HEART LESSON IN EOSINOPHILIC GRANULOMATOSIS WITH POLYANGIITIS}

D. Rednic ${ }^{1}$, R. Rancea ${ }^{2}$, F. Cozma ${ }^{1}$, L. Damian ${ }^{1}$, L. Muntean ${ }^{1,3}$,

M.M. Tamas ${ }^{1,3}$, S.-P. Simon ${ }^{1,3}$, I. Felea ${ }^{1}$, S. Rednic ${ }^{1,3}$. ${ }^{1}$ Rheumatology, Clinical County Emergency Hospital; ${ }^{2}$ Cardiology, Heart Institute "Niculae Stancioiu";

3 "Iuliu Hatieganu" University of Medicine and Pharmacy, Cluj-Napoca, Romania

Background: Eosinophilic granulomatosis with polyangiitis (EGPA) is a disorder characterised by systemic small vessel vasculitis, that occurs typical in patients with a positive history of late-onset asthma and allergic rhinitis. Cardiac involvement is the major cause of mortality. There is required a standardised method to assess cardiac involvement in EGPA

Objectives: To assess the clinical and imagistic caracteristics of EGPA patients with cardiac involvement in a tertiary referral hospital.

Methods: Fourteen patients (pts) with EGPA were retrospectively analysed between 2010-2016, in Rheumathology Departament in Cluj-Napoca. All patients were screened for cardiac involvement by electrocardiogram (ECG) and cardiac ultrasonography (CUS). Cardiac involvement was defined as follows: ventricular hypertrophy, kinetic abnormalities, valvulopathy, pericardial effusion or diastolic dysfunction. Cardiac magnetic resonance (CMR) and coronarographie was assessed in 4 pts with ECG or CUS abnormalities.

Results: Characteristics of EGPA patients is detailed in table 1. Six out of 14 pts had cardiac involvement (table 2). Only one patient was symptomatic CMR abnormalities were: endocardial fibrosis in 2 pts, subepicardial inflammation

Table 1. Demographic caracteristic of EGPA patients. EGPA, eosinophilic granulomatosis with polyangiitis; Cardiac, patients with cardiac involvement, Non cardiac, pacients without cardiac involvement; ANCA, anti-neutrophil cytoplasmatic antibody

\begin{tabular}{lccc}
\hline Characteristics of EGPA Patients & $\mathrm{n}=14$ & Cardiac $(\mathrm{n}=6)$ & Non cardiac $(\mathrm{n}=8)$ \\
\hline Age at onset, $\mathrm{yr} \pm \mathrm{SD}$ & $54.57 \pm 9.7$ & $53 \pm 12.19$ & $55.75 \pm 8.04$ \\
Female, $\mathrm{n}$ & 8 & 4 & 4 \\
History of astm, $\mathrm{n}$ & 7 & 3 & 4 \\
ANCA (+), $\mathrm{n}$ & 5 & 3 & 2 \\
Organ involvement, $\mathrm{n}$ & & & \\
Renal & 1 & 4 & 0 \\
Pulmonary & 9 & 5 & 5 \\
Neurologic & 10 & 0 & 1 \\
Ophtalmic & 1 & 1 & 5 \\
Skin & 6 & &
\end{tabular}

Table 2. Electrocardiographic and echocardiographic findings in EGPA patients with cardiac involvement

Electrocardiographic and echocardiographic findings

Conduction disorders

Ischemia in inferior leads, $\mathrm{n}$

Pericardial effusion, $n$

Kinetic abnormalities, $\mathrm{n}$

Septal thickening, $n$

Mitral insufficiency, $\mathrm{n}$

Diastolic dysfunction, $\mathrm{n}$

$n=6$
2
1
4
3
3
1
4

